ESAIM: Proceedings, Vol. 8, 2000, 95-105

CONTRÔLE DES SYSTÈMES GOUVERNÉS

PAR DES ÉQUATIONS AUX DÉRIVÉES PARTIELLES

http://www.emath.fr/proc/Vol.8/

\title{
On exact controllability of generic trees
}

\author{
Günter Leugering* $\quad$ Enrique Zuazua ${ }^{\dagger}$
}

\begin{abstract}
We consider a divining rod as an exemplaric network of elastic homogeneous strings. If the lengths of the strings are rationally dependent, it is known that even approximate controllability by a single boundary control fails, whenever the other two simple nodes satisfy the same boundary condition. In this paper we give a positive answer to the question whether exact controllability for some class of initial/final data holds, if the individual lengths of the strings are no longer rationally dependent. In order to do this, we resort to a special class of algebraic numbers, namely Roth's class. However, we do not use Fourier series expansions but rather d'Alembert's formula to analyze the propagation of the effect of the controllers along the network.
\end{abstract}

\section{Introduction}

The problem of controllability of networks of elastic strings or beams is under investigation since about ten years. See Lagnese, Leugering and Schmidt [6], as a general reference also for multi-link membrane and plate problems. As we do not intend to overburden these notes with clumsy notations in order to describe the mathematical details of general network control problems, we choose to present the known results in a somewhat informal way resorting to typical graph-theoretic notions which are, however, self explanatory. We do this for string-networks, only:

i). Let $G$ be a tree of $1-d$-elastic bodies (prestreched strings). If the root is clamped and all leaves are controlled, either using $H^{1}(0, T)$-Dirichlet - or $L^{2}(0, T)$ Neumann controls, then natural finite energy data are exactly controllable in a finite time which is twice the length of the longest path from the root to a controlled leaf. $([6])$.

ii). Let $G$ be as in i). If there is an uncontrolled leaf which satisfies homogeneous Dirichlet conditions (together with the root), and if the path between these nodes consists of strings with their optical lengths being rationally dependent, then even approximate controllability fails to hold. ([6]).

iii). Let $G$ be a graph containing a circuit. If the strings constituing that circuit have rationally dependent optical lengths, then approximate controllability fails, regardless of how many controls are imposed on the network. ([6]).

\footnotetext{
*Fakultät für Mathematik und Physik, Universität Bayreuth, D-95440 Bayreuth, Germany. email: leugering@uni-bayreuth.de

${ }^{\dagger}$ Departamento de Matematica Aplicada, Universidad Complutense de Madrid, 28040 Madrid, Spain. e-mail: zuazua@eucmax.sim.ucm.es
} 
There are also examples of exactly controllable rooted networks where all but one of the leaves (other than the root) are controlled, while the uncontrolled leaf satisfies Neumann conditions (see [7],[8]). Similar statements are true for networks of Timoshenko beams and symmetric linear hyperbolic systems on graphs. On the other side, very little is known for networks of Euler-Bernoulli or Rayleigh-beams. The only positive results available for Euler-Bernoulli-beam networks are: exact controllability for star-graphs, if all leaves are controlled (slide and turn controls). See Leugering and Schmidt [9]. Very recently Dekoninck and Nicaise [4] have established some results for a class of scalar beam models. It is striking that the remarkably simple problem of exact controllability of a planar carpenter's square is still open, even though there is strong numerical evidence that this situation is exactly controllable; see Briffaut [3].

Let us return to the string problems. Indeed, the survey of results just given is somehow not very satisfactory - the results appear fragmentary. In particular, it is striking that either exact controllability can be shown to hold (by various methods) or not even approximate controllability can be achieved. It appears that the negative results are typically obtained on the base of the investigation of 'aberrant' eigenelements, namely those which admit zeros where an observation has to take place. Whenever such aberrant eigenmodes were shown to not exist, one was able to show exact controllability. In this sense it seemed that in the case of $1-d$-wave equations controllability was essentially a matter of checking eigenmodes, hence, a 'finite-dimensional' problem. In fact that problem was raised by C. Bardos. In these notes we show that, indeed, in generic situations to be defined below, when exact controllability in finite energy fails to hold, we might have exact controllability in smoother spaces.

In particular, we will show that a star-graph, which we take as an 'atom' of general graphs, is exactly controllable from the root in a smooth energy space, if all individual lengths of the strings are mutually in a certain class of algebraic numbers, namely Roth's class. See Stolarsky [12] for the theory of diophantine approximations. At the first glance such a result appears to be purely academic: it serves to answer a long-standing open problem. The question is whether there is also any practical substance to it. Certainly, an engineer is not supposed to produce mechanical elements with a length in Roth's class. Neither is a numerical analyst able to produce reliable numerical evidence, when it comes down to such numbers - simply as they are not available on the computer. Still, we think that results in this direction are useful even for practical reasons, in that we follow the arguments of network control engineers who have coined the notion of structural controllability for precisely this situation. Our approach, therefore, can be seen as a first step towards an infinite dimensional extension of that theory.

After this introductory remarks, we want to describe the precise mathematical problem to be studied.

\section{Remarks on a two-link serial string problem}

We consider a string of length 1 clamped at $x=0$ and $x=1$. Assume that a control is applied at an internal point $x=a$, by applying a force there. State- 
ments on controllability are established by investigation of the adjoint homogeneous problem. As an introduction to multi-link systems, we view this problem as a two-link system with control at a multiple node. We introduce displacements $\varphi_{i}(x, t), x \in\left(0, l_{i}\right), t \in(0, T), l_{1}=a, l_{2}=1-a$ such that $\varphi_{1}(0, t), \varphi_{2}(0, t)$ correspond to the common displacement at $a$, while $\varphi_{1}\left(l_{1}, t\right), \varphi_{2}\left(l_{2}, t\right)$ are the mutual values at 0 , and 1 , respectively. We thus direct the strings from the junction to the ends. With this convention the system to look at is given by

$$
\begin{cases}\ddot{\varphi}_{i}=\varphi_{i}^{\prime \prime}, x \in\left(0, l_{i}\right), i=1,2, & t \in(0, T), \\ \varphi_{i}\left(l_{i}, t\right)=0, i=1,2, & t \in(0, T), \\ \varphi_{1}(0, t)=\varphi_{2}(0, t), & t \in(0, T), \\ \varphi_{1}^{\prime}(0, t)+\varphi_{2}^{\prime}(0, t)=0, & t \in(0, T), \\ \varphi_{i}(x, 0)=\varphi_{i 0}(x), \dot{\varphi}_{i}(x, 0)=\varphi_{i 1}(x), & x \in\left(0, l_{i}\right) .\end{cases}
$$

The observability problem requires then to retrieve, as much as possible, information from the observation of $\varphi_{i}(0, t), t \in(0, T)$.

The very first and basic question is whether $\varphi_{i}(0, t) \equiv 0, t \in(0, T)$ implies $\varphi_{i}(\cdot) \equiv$ $0, i=1,2$. Obviously, for an eigenfunction $\psi$, represented locally by $\psi_{i}(x)$ such that

$$
\left\{\begin{array}{l}
\psi_{i}^{\prime \prime}=-\lambda^{2} \psi_{i}, x \in\left(0, l_{i}\right), i=1,2, \\
\psi_{i}\left(l_{i}\right)=0, \psi_{1}(0)=\psi_{2}(0)=0, \\
\psi_{1}^{\prime}(0)+\psi_{2}^{\prime}(0)=0
\end{array}\right.
$$

$\varphi_{i}(x, t)=\cos (\lambda t) \psi_{i}(x)$ solves $(2.1)$ with $\varphi_{i}(x, 0)=\psi_{i}(x), \dot{\varphi}_{i}(x, 0)=0$. But solving (2.2) implies $l_{1} / l_{2} \in \mathbb{Q}$. This is essentially the situation in ii) of the introduction. See also Avdonin [1], Theorem V.1.7.(b).

If is easy to refine this argument and to deal with a string and a number of intermediate points $x_{i} i=1 ; \ldots, n$. In fact, such a problem can easily be recast into the framework of serial strings as in (2.1). One can thereby construct a non-trivial solution $u(x, t)$ of the wave equation on $(0,1)$ with Dirichlet boundary data, such that

$$
u\left(\frac{k}{n}, t\right)=0, \quad k=\{0,1,2, \ldots, n\}
$$

A similar argument covers networks of strings; see [6].

Even if all eigenfunctions do not vanish at the point $x=0$ of in-span control (in the case of the two-link system above this is true if $l_{1}=a \in(0,1) \backslash \mathbb{Q}$ ), exact controllability with respect to the natural energy space (i.e. $E_{0}:=H^{1}(0,1) \times$ $L^{2}(0,1)$ ) does not hold for any $T$, while spectral controllability holds for $T \geq 2$. See [1] Theorem V.1.7 (c), (d). The latter implies that for $l_{1}$ irrational approximate controllability within $E_{0}$ holds.

In order to proceed to positive results one has to consider irrational numbers which are 'badly approximable by rationals' in a precise sense. Namely, we consider algebraic numbers $a \in(0,1) \backslash \mathbb{Q}$ with degree $d(a) \geq 2$. It has been shown by Roth (see Stolarsky [12], Thm. 6.1.1, p. 98) that for each $\epsilon>0$ there is some $c(a, \epsilon)>0$ such that

$$
\left|a-\frac{p}{q}\right|>\frac{c(a, \epsilon)}{q^{2+\epsilon}}
$$


for all rationals $\frac{p}{q}$. Note that for algebraic numbers of degree $d(a)=2$, Liouville's theorem asserts that (2.4) holds with $\epsilon=0$, see Stolarsky [12]. The point is that by Roth's theorem all non-rational algebraic numbers satisfy some inequality (2.4), such that for each $\epsilon>0$ a class $\mathcal{A}_{\epsilon}$ of such numbers exists satisfying (2.4) with $c$ independent of $a$. This set $\mathcal{A}_{\epsilon}$ has full Lebesgue measure. Resorting to Liouville's result, Khapalov [5] has shown that, indeed, the space of controllable data has to be smoother by 1 degree than if the observation point would have been taken as a boundary point. This has also been noted by Masrour and Zuazua [11], in an unpublished communication. See also Ammari and Tucsnak [2] and Tucsnak [13] for related results, also concerning Euler-Bernoulli beams.

\section{On Fourier-mulitipliers}

We proceed to show that in the 'generic' case where the 'optical lenghts' are rationally independent in the sense of Roth, we obtain exact controllability in an appropriate energy space. Instead of using a modal approach, we want to pursue our analysis on the base of propagation properties. We, therefore, consider the mappings

$$
\left(D_{x}^{ \pm} z\right)(t):=z(t+x) \pm z(t-x), \quad D_{x}^{ \pm}: L^{2}(a, b) \rightarrow L^{2}(a+x, b-x)
$$

which naturally appear in d'Alembert's solution of the 1-d wave equation. We consider two situations: one on the entire real line, and one on a finite time interval. With respect to controllability results we will resort to the second scenario. However, for the sake of completeness and clarity we also treat the first case, which, indeed, has been announced in [11]:

Lemma 1. Let $f$ be a distribution and $a \in \mathcal{A}_{\epsilon}, d(a) \geq 2$. Then $D_{a}^{-} f, D_{1-a}^{-} f \in$ $L^{2}(\mathbb{R})$ imply $f \in H^{-1-\epsilon}(\mathbb{R})$.

Proof. We take Fourier transforms of $v:=D_{a}^{-} f$ and $w:=D_{1-a}^{-} f$

$$
\begin{aligned}
\hat{v}(\omega) & =\left(e^{i \omega a}-e^{-i \omega a}\right) \hat{f}(\omega)=2 i \sin (\omega a) \hat{f}(\omega), \\
\hat{w}(\omega) & =\left(e^{i \omega(1-a)}-e^{-i \omega(1-a)}\right) \hat{f}(\omega)=2 i \sin (\omega(1-a)) \hat{f}(\omega) .
\end{aligned}
$$

Now $\hat{v}, \hat{w} \in L^{2}(\mathbb{R})$, hence

$$
\int_{\mathbb{R}}\{2-\cos (2 \omega a)-\cos (2 \omega(1-a))\}|\hat{f}(\omega)|^{2} d \omega<\infty .
$$

We consider the set $E=\cup_{l \in Z \backslash 0}\left\{\pi \frac{\ell}{1-a}+\left[-\frac{\rho}{\ell^{1+\epsilon}}, \frac{\rho}{\ell^{1+\epsilon}}\right]\right\}, \rho>0$.

There are two cases to be considered: a.) $\omega \in E$ and b.) $\omega \notin E$. The idea is that in either case the quantitiy $c(\omega)=2-\cos (2 \omega a)-\cos (2 \omega(1-a))$ can be bounded below by $\frac{c}{\omega^{2(1+\epsilon)}}$. Modulo a (smooth) function of local support around $\omega=0$, we then have this estimate on entire $\mathbb{R}$, which in turn gives the result. 
a.) $\omega \notin E, \omega \neq 0$ implies $|\cos (2 \omega(1-a))| \leq \cos \left(\frac{2(1-a) \rho}{\ell^{1+\epsilon}}\right)$. Hence,

$$
1-\cos (2 \omega(1-a)) \geq 1-\cos \left(\frac{2(1-a) \rho}{\ell^{1+\epsilon}}\right)=2 \sin ^{2}\left(\frac{(1-a) \rho}{\ell^{1+\epsilon}}\right) \geq \frac{\rho^{2}}{\ell^{2(1+\epsilon)}},
$$

for $\rho>0$ sufficiently small. It follows that $c(\omega) \geq 1-\cos (2 \omega(1-a)) \geq \frac{\rho^{2}}{\ell^{2(1+\epsilon)}}$.

b.) Now assume $\omega \in E$ : then for some $\ell$ we have the representation

$$
\omega=\pi \frac{\ell}{1-a}+x, \quad|x| \leq \frac{\rho}{\ell^{1+\epsilon}},
$$

from which it follows that $2 a \omega=2 \pi \ell \frac{a}{1-a}+a x$. For any $k$ we have

$$
\left|2 \pi \ell \frac{a}{1-a}-2 \pi k\right|=2 \pi \ell\left|\frac{a}{1-a}-\frac{k}{\ell}\right| \geq 2 \pi \ell \frac{c_{\epsilon}}{\ell^{2+\epsilon}}=\frac{c_{\epsilon} 2 \pi}{\ell^{1+\epsilon}}
$$

by Roth's lemma (Note $a \in \mathcal{A}_{\epsilon} \Leftrightarrow \frac{a}{1-a} \in \mathcal{A}_{\epsilon}$ ). Now

$$
|2 a \omega-2 \pi k|=\left|2 \pi \ell \frac{a}{1-a}-2 \pi k+a x\right| \geq \frac{2 \pi c_{\epsilon}}{\ell^{1+\epsilon}}-|a| \frac{\rho}{\ell^{1+\epsilon}} \geq \frac{\alpha}{\ell^{1+\epsilon}},
$$

for some positive $\alpha$, as long as $\rho$ is sufficiently small. But again, then $\cos (a \omega) \leq$ $\cos \left(\frac{\alpha}{\ell^{1+\epsilon}}\right)$ from which it follows that $1-\cos (2 a \omega) \geq \frac{\beta}{\ell^{2(1+\epsilon)}}$ for some positive $\beta$ and, hence, $c(\omega) \geq \frac{\min \left(\beta, \rho^{2}\right)}{\ell^{2(1+\epsilon)}}$. Note that $\alpha$ and hence $\beta$ are independent of $\ell$ and can be made small by choosing $\rho$ small.

We conclude that on all of $\mathbb{R}$, but a neighbourhood of zero, we have with $\omega=\mathcal{O}(\ell)$ :

$$
|2-\cos (2 \omega a)-\cos (2 \omega(1-a))| \geq \frac{c}{1+|\omega|^{2(1+\epsilon)}}, \quad \text { on } \mathbb{R} \backslash[-\delta, \delta] .
$$

This proves $f \in H^{-1-\epsilon}(\mathbb{R})$ as stated.

Remark 1. If $d(a)=2$ in Lemma 1, then according to Liouville's theorem $\mid 2 \pi \ell \frac{a}{1-a}-$ $2 \pi k \mid \geq \frac{c 2 \pi}{\ell}$ for any $k$. Thus, upon deleting $\epsilon$ in the proof of the lemma we arrive at the conclusion that $f \in H^{-1}(\mathbb{R})$.

Obviously, it is enough to assume that $f(x)-f(x+1)$ and $f(x)-f(x+a)$ are in $L^{2}(\mathbb{R})$ in order to arrive at the same conclusion. We took the setting with the mappings $D_{a}^{-}$for the sake of symmetry with the case, where we consider a finite time interval.

Lemma 2. Let $f$ be a 2-periodic distribution on $[-1,1]$ and $a \in \mathcal{A}_{\epsilon}, d(a) \geq 2$. Then $D_{a}^{-} f \in L^{2}(-(1-a), 1-a), D_{1-a}^{-} f \in L^{2}(-a, a)$ imply $f \in H^{-1-\epsilon}(-1,1)$, i.e. there exists a positive constant $C$, depending on only on $\epsilon$, such that

$$
\|f\|_{H^{-1-\epsilon}(-1,1)} \leq C\left\{\left\|D_{a}^{-} f\right\|_{L^{2}(-(1-a), 1-a)}+\left\|D_{1-a}^{-} f\right\|_{L^{2}(-a, a)}\right\} .
$$


Proof. Let $f(t)=\sum_{k \in \mathbb{Z}} \hat{f}(k) e^{i k \pi t}$ be the Fourier representation of $f$ on the interval $[-1,1]$. We have

$$
\begin{aligned}
h(t) & :=D_{a}^{-} f(t)=\sum_{k \in \mathbb{Z}} \hat{f}(k) e^{i k \pi t}\left(e^{i k \pi a}-e^{-i k \pi a}\right) \\
& =2 i \sum_{k \in \mathbb{Z}} \hat{f}(k) \sin (k \pi a) e^{i k \pi t}
\end{aligned}
$$

and similarly

$$
\begin{aligned}
g(t) & :=D_{1-a}^{-} f=-2 i \sum_{k \in \mathbb{Z}} \hat{f}(k) \sin (k \pi a) \cos (k \pi) e^{i k \pi t} \\
& =2 i \sum_{k \in \mathbb{Z}}(-1)^{k+1} \hat{f}(k) \sin (k \pi a) e^{i k \pi t} .
\end{aligned}
$$

Moreover, shifting $h(t)$ by $a$, i.e. $\tilde{h}(t):=h(t+a)$ and $g(t)$ by $-(1-a)$, i.e. $\tilde{g}(t):=$ $g(t-(1-a))$, we realize that $\tilde{g}(t)=-\tilde{h}(t)$. But

$$
\begin{aligned}
\|\tilde{h}\|_{L^{2}(-1,1-2 a)} & =\int_{-1}^{1-2 a}|h(t+a)|^{2} d t=\int_{-(1-a)}^{1-a}|h(t)|^{2} d t, \\
\|\tilde{g}\|_{L^{2}(1-2 a, 1)} & =\int_{1-2 a}^{1}|g(t-(1-a))|^{2} d t=\int_{-a}^{a}|g(t)|^{2} d t .
\end{aligned}
$$

This implies

$$
\begin{aligned}
& \left\|D^{-} f_{1-a}\right\|_{L^{2}(-a, a)}^{2}+\left\|D_{a}^{-} f\right\|_{L^{2}(-(1-a), 1-a)}^{2} \\
& =\int_{-(1-a)}^{1-a}|h(t)|^{2} d t+\int_{-a}^{a}|g(t)|^{2} d t=\int_{-1}^{1-2 a}|\tilde{h}(t)|^{2} d t+\int_{1-2 a}^{1}|\tilde{g}(t)|^{2} d t \\
& \quad=\|\tilde{h}\|_{L^{2}(-1,1)}^{2}=\sum_{k \in \mathbb{Z}}|\hat{f}(k)|^{2}\left|\sin ^{2}(k \pi a)\right| .
\end{aligned}
$$

But

$$
|\sin (k \pi a)|=\frac{|\sin (k \pi a-\pi \ell)|}{|k \pi a-\pi \ell|} \geq c|k a-\ell|=c k\left|a-\frac{k}{\ell}\right| \geq \frac{c k}{k^{2+\epsilon}}
$$

and the desired result follows.

Remark 2. i) It is clear from the proof that one can directly assume

$$
|\sin (k \pi a)| \geq \frac{c}{k^{s}}, \quad \forall k \in \mathbb{N}
$$

for some positive $c, s$, in order to conclude $f \in H^{-s}(\mathbb{R})$. 


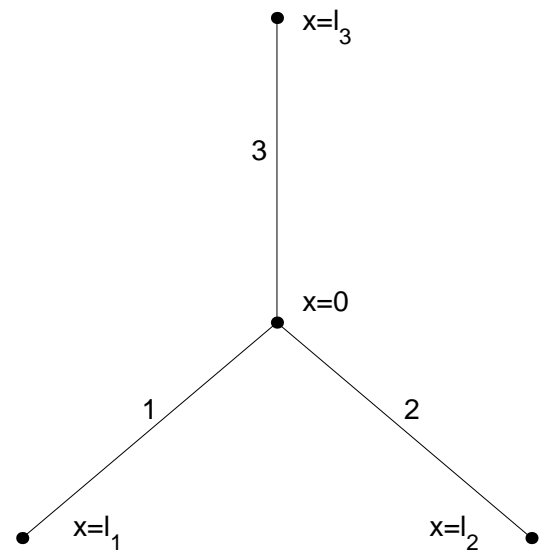

Figure 1: The star network of strings at the reference configuration

ii) If $d(a)=2$ in Lemma 2 then we can take $\epsilon=0$ as in Remark 1.

iii) It is known (e.g. from [1] Theorem V.1.7. (c)) that as soon as a is irrational (hence $\sin (k \pi a) \neq 0, \forall k$ ) the system is approximately controllable ( indeed spectrally controllable). In that case (and the case above) spaces of controllable data can be constructed in principle using suitable weights in the Forier series expansions. We would like to stress, however, that we do not rely on eigenfunctions and eigenvalues and, hence, on the series representation of solutions.

\section{A divining rod is controllable from the root}

We consider three strings coupled at one point, say at $x=0$, extending in different directions. For simplicity we assume that the reference configuration is in the plane and that the strings are subject to scalar displacements out of the plane, i.e., there is no in-plane motion. The $3-d-$ situation could be considered as well. Again, for simplicity only, we take all density and stiffness parameters equal to unity. That is, we concentrate on the effect of having different lengths denoted by $l_{i}, i=1,2,3$. We first write down the forward controllability problem.

$$
\begin{cases}\ddot{u}_{i}=u_{i}^{\prime \prime}, x \in\left(0, l_{i}\right), t \in(0, T), & i=1,2,3 \\ u_{1}\left(l_{1}, t\right)=v(t), u_{i}\left(l_{i}, t\right)=0, i=2,3, & t \in(0, T), \\ u_{i}(0, t)=u_{j}(0, t), i \neq j, i, j=1,2,3, & t \in(0, T), \\ \sum_{i=1}^{3} u_{i}^{\prime}(0, t)=0, & t \in(0, T), \\ u_{i}(\cdot, 0)=u_{i 0}, \dot{u}_{i}(\cdot, 0)=u_{i 1}, & x \in\left(0, l_{i}\right), i=1,2,3 .\end{cases}
$$

See figure 1 for this particular set-up.

Remark 3. System (4.6) is wellposed in $E_{0}$ with $v \in H_{0}^{1}(0, T)$, and, by transposition, wellposedness extends to $E_{-1}$ for $v \in L^{2}(0, T)$. See Lagnese, Leugering and 
Schmidt [6]. In this context $E_{0}=V \times H$, where $H=\prod_{i=1}^{3} L^{2}\left(0, l_{i}\right)$ and

$$
V=\left\{u=\left(u_{i}\right)_{i=1,2,3} \mid u_{i} \in H^{1}\left(0, l_{i}\right) u_{i}(0)=u_{j}(0), u_{i}\left(l_{i}\right)=0, i, j=1,2,3\right\},
$$

while $E_{-1}=H \times V^{\star}$.

The controllability problem reads as follows:

Problem (EC): Let $T>0$ be sufficiently large. Given $\left(u_{0}, u_{1}\right) \in E_{-1}$, does there exist a control $v \in L^{2}(0, T)$ such that the solution $u$ of (4.6) satisfies

$$
u_{i}(\cdot, T)=\dot{u}_{i}(\cdot, T) \equiv 0 \quad i=1,2,3 ?
$$

Remark 4. We know that problem (EC) does not have an affirmative answer in case $l_{2}=l_{3}$ (or even $l_{2}, l_{3}$ rationally dependent). In these cases the space of controllable data is not even dense in $E_{-1}$ where $E_{-s}:=H^{-s+1} \times H^{-s}$.

As always, we are going to investigate the adjoint problem:

$$
\begin{cases}\ddot{\varphi}_{i}=\varphi_{i}^{\prime \prime}, x \in\left(0, l_{i}\right), i=1,2,3, & t \in(0, T), \\ \varphi_{i}\left(l_{i}, t\right)=0, i=1,2,3, & t \in(0, T), \\ \varphi_{i}(0, t)=\varphi_{j}(0, t), i \neq j=1,2,3, & t \in(0, T), \\ \sum_{i=1}^{3} \varphi_{i}^{\prime}(0, t)=0, & t \in(0, T), \\ \varphi_{i}(\cdot, 0)=\varphi_{i 0}, \dot{\varphi}_{i}(\cdot, 0)=\varphi_{i 1}, & x \in\left(0, l_{i}\right), i=1,2,3 .\end{cases}
$$

As is well-known, the observability estimate associated with (EC) is

$$
\sum_{i=1}^{3} \int_{0}^{l_{i}}\left[\left|\varphi_{i, 1}(x)\right|^{2}+\left|\varphi_{i, 0}^{\prime}(x)\right|^{2}\right] d x \leq C \int_{0}^{T}\left|\varphi_{1}^{\prime}\left(l_{1}, t\right)\right|^{2} d t .
$$

But, as we stated in the introduction, an inequality like (4.8) fails to hold for $l_{2} / l_{3} \in \mathbb{Q}$. In fact, in this case there are nontrivial solutions of (4.7) such that $\varphi_{1}^{\prime}\left(l_{1}, t\right)=0$ for all $t$. Thus even approximate controllability fails. The question then should be stated as follows: when $l_{2} / l_{3} \notin \mathbb{Q}$ is there any energy space $E_{-s}, s>0$ for which an estimate (4.8) would be true?

We proceed as follows. If we view $\varphi^{\prime}\left(l_{1}, t\right)$ as the only input, then string 1 evolves freely until $t=l_{1}$. Interchanging the role of $x$ and $t$ one obtains the estimate:

$$
\forall x \in\left(0, l_{1}\right): \int_{l_{1}-x}^{T-l_{1}+x}\left[\left|\dot{\varphi}_{1}(x, t)\right|^{2}+\left|\varphi_{1}^{\prime}\left(l_{1}, t\right)\right|^{2}\right] d t \leq \int_{0}^{T}\left|\varphi_{1}^{\prime}\left(l_{1}, t\right)\right|^{2} d t
$$

which we evaluate at $x=0$

$$
\int_{l_{1}}^{T-l_{1}}\left[\left|\dot{\varphi}_{1}(0, t)\right|^{2}+\left|\varphi_{1}^{\prime}(0, t)\right|^{2}\right] d t \leq \int_{0}^{T}\left|\varphi_{1}^{\prime}\left(l_{1}, t\right)\right|^{2} d t .
$$

Using the transmission conditions $(4.7)_{3,4}$ we obtain

$$
\begin{aligned}
\int_{l_{1}}^{T-l_{1}}\left\{\left|\dot{\varphi}_{2}(0, t)\right|^{2}+\left|\dot{\varphi}_{3}(0, t)\right|^{2}+\left|\varphi_{2}^{\prime}(0, t)+\varphi_{3}^{\prime}(0, t)\right|^{2}\right\} & d t \\
& \leq 2 \int_{0}^{T}\left|\varphi_{1}^{\prime}\left(l_{1}, t\right)\right|^{2} d t .
\end{aligned}
$$


Again, if in (4.11) instead of the square of the sum $\varphi_{2}^{\prime}(0, t)+\varphi_{3}^{\prime}(0, t)$ we would have the sum of squares, then we could use energy estimates, locally in each individual string. The main question then is, just how much energy can be seen from observations at the end of string number 1 ?

We change the interval from $\left(l_{1}, T-l_{1}\right)$ to $\left(0, T-2 l_{1}\right)=(0, \tilde{T})$. Define the common values at $x=0$ as $f(t)$ as well as

$$
g(t):=\sum_{i=2}^{3} \varphi_{i}^{\prime}(0, t)=-\varphi_{1}^{\prime}(0, t), \quad h(t):=\varphi_{2}^{\prime}(0, t) .
$$

Then, according to (4.10), (4.11) we have

$$
\left\{\begin{aligned}
f(\cdot)=\varphi_{1}(0, \cdot)=\varphi_{2}(0, \cdot) & \in H^{1}(0, \tilde{T}) \\
g(\cdot)=\varphi_{2}^{\prime}(0, \cdot)+\varphi_{3}^{\prime}(0, \cdot)=h(\cdot)+\varphi_{3}^{\prime}(0, \cdot) & \in L^{2}(0, \tilde{T})
\end{aligned}\right.
$$

With these data we can solve the wave equation by using d'Alembert's formula

$$
\left\{\begin{aligned}
\varphi_{2}(x, t) & =\frac{1}{2}(f(t+x)+f(t-x))+\frac{1}{2} \int_{t-x}^{t+x} h(s) d s \\
\varphi_{3}(x, t) & =\frac{1}{2}(f(t+x)+f(t-x))+\frac{1}{2} \int_{t-x}^{t+x}(g-h)(s) d s .
\end{aligned}\right.
$$

But, taking into account the Dirichlet boundary condition $(4.7)_{2}$ we obtain

$$
\begin{aligned}
\varphi_{2}\left(l_{2}, t\right) & =\frac{1}{2}\left(f\left(t+l_{2}\right)+f\left(t-l_{2}\right)\right)+\frac{1}{2} \int_{t-l_{2}}^{t+l_{2}} h(s) d s=0, \\
\varphi_{3}\left(l_{3}, t\right) & =\frac{1}{2}\left(f\left(t+l_{3}\right)+f\left(t-l_{3}\right)\right)+\frac{1}{2} \int_{t-l_{3}}^{t+l_{3}}(g(s)-h(s)) d s=0
\end{aligned}
$$

which upon differentiation with respect to $t$ gives

$$
\left\{\begin{aligned}
\dot{\varphi}_{2}\left(l_{2}, t\right)=\frac{1}{2}\left\{\dot{f}\left(t+l_{2}\right)+\right. & \dot{f}\left(t-l_{2}\right) \\
& \left.+h\left(t+l_{2}\right)-h\left(t-l_{2}\right)\right\}=0 \\
\dot{\varphi}_{3}\left(l_{3}, t\right)=\frac{1}{2}\left\{\dot{f}\left(t+l_{3}\right)+\right. & \left.\dot{f}\left(t-l_{3}\right)\right)+g\left(t+l_{3}\right)-g\left(t-l_{3}\right) \\
& \left.-\left(h\left(t+l_{3}\right)-h\left(t-l_{3}\right)\right)\right\}=0 .
\end{aligned}\right.
$$

But $\dot{f}, g \in L^{2}(0, \tilde{T})$, so that (4.14) implies

$$
\left\{\begin{array}{l}
D_{\ell_{2}}^{-} h(t)=h\left(t+l_{2}\right)-h\left(t-l_{2}\right) \in L^{2}\left(l_{2}, \tilde{T}-l_{2}\right) \\
D_{\ell_{3}}^{-} h(t)=h\left(t+l_{3}\right)-h\left(t-l_{3}\right) \in L^{2}\left(l_{3}, \tilde{T}-l_{3}\right) .
\end{array}\right.
$$

We have $T=2\left(l_{1}+l_{2}+l_{3}\right)$ and $\tilde{T}=2\left(l_{2}+l_{3}\right)$. Therefore, $l_{3}=\frac{\tilde{T}}{2}-l_{2}$ and $\tilde{T}-l_{3}=\frac{\tilde{T}}{2}+l_{2}$. We set $a=l_{2}$ and write instead of (4.15)

$$
D_{a}^{-} h \in L^{2}(a, \tilde{T}-a), \quad D_{\frac{\tilde{T}}{2}-a}^{-} h \in L^{2}\left(\frac{\tilde{T}}{2}-a, \frac{\tilde{T}}{2}+a\right)
$$


We now translate the time intervals $(a, \tilde{T}-a),\left(\frac{\tilde{T}}{2}-a, \frac{\tilde{T}}{2}+a\right)$ by $-\frac{\tilde{T}}{2}$ into $\left(-\left(\frac{\tilde{T}}{2}-\right.\right.$ $\left.a), \frac{\tilde{T}}{2}+a\right)$ and $(-a, a)$, respectively. Then we may apply Lemma 2 on the time interval $\left(-\frac{\tilde{T}}{2}, \frac{\tilde{T}}{2}\right)$ instead of $(-1,1)$. Thereby we have proved our main result.

Theorem 1. Let $T \geq 2\left(l_{1}+l_{2}+l_{3}\right), l_{2} / l_{3} \in \mathcal{A}_{\epsilon}$ be given. Then there exists a constant $C$ such that

$$
\sum_{i=1}^{3}\left\|\varphi_{i}(\cdot, 0)\right\|_{H^{-\epsilon}}^{2}+\left\|\dot{\varphi}_{i}(\cdot, 0)\right\|_{H^{-1-\epsilon}}^{2} \leq C \int_{0}^{T}\left|\varphi^{\prime}\left(l_{1}, t\right)\right|^{2} d t .
$$

In other words, for any initial data $\left(u_{0}, u_{1}\right)$ with

$$
u_{0} \in V, u_{i 0} \in H^{1+\epsilon}\left(0, l_{i}\right), u_{i 1} \in H^{\epsilon}\left(0, l_{i}\right),
$$

there is a control $v \in L^{2}(0, T)$ such that the solution $u$ of (4.6) satisfies

$$
u_{i}(\cdot, T) \equiv \dot{u}_{i}(\cdot, T) \equiv 0 \quad i=1,2,3 .
$$

Remark 5. i) As we rely on Lemma 2, we may use Remark 2 and state that for $d\left(l_{2} / l_{3}\right)=2$ the result of the theorem holds with $\epsilon=0$. Moreover, for irrational $l_{2} / l_{3}$ we obtain approximate controllability within the natural energy space and the same time interval.

ii) Extensions of this result to arbitrary star-graphs and general trees also including beam elements are under way.

iii.) Using Russell's principle relating controllability of hyperbolic and parabolic problems, our results imply corresponding null-controllability results for parabolic networks for any control time.

\section{References}

[1] S. Avdonin and S. Ivanov. Families of exponentials, Cambridge University Press 1995.

[2] K. Ammari and M. Tucsnak. Stabilization of Euler-Bernoulli beams by means of a pointwise feedback force. Preprint, 1999.

[3] J.-S. Briffaut. Méthodes numériques pour le contrôle et la stabilisation rapide des grandes structures flexibles, PhD-thesis at École Nationale des Ponts et Chaussées, 1999.

[4] B. Dekoninck and S. Nicaise. Control of networks of Euler-Bernoulli beams, 1999. ESAIM-COCV, 4: 57-81, 1999.

[5] A. G. Khapalov. Interior point control and observation for the wave equation. Abstract and Applied Analysis, 1(2): 219-236, 1996. 
[6] J.E. Lagnese, G. Leugering, and E.J.P.G. Schmidt. Modeling, Analysis and Control of Dynamic Elastic Multi-Link Structures. Birkhäuser, Boston-BaselBerlin, 1994.

[7] G. Leugering. Reverberation analysis and control of networks of elastic strings. In E. Casas, editor, Control of Partial Differential Equations and Applications, pages 193-206. Marcel Dekker, 1996.

[8] G. Leugering. On dynamic domain decomposition of controlled networks of elastic strings and joint masses. In F. Kappel, editor, Control of Distributed Parameter Systems, ISNM 126, pages 199-205. Birkhäuser, 1998.

[9] G. Leugering and E.J.P.G. Schmidt. On the control of networks of vibrating strings and beams. In Proceedings of the 28th IEEE Conference on Decision and Control, volume 3, pages 2287-2290. IEEE 1989.

[10] G. Leugering and E. Zuazua. On the problem of exact and approximate controllability of generic networks of elastic strings. In preparation, 1999.

[11] T. Masrour and E. Zuazua. Observation curviligne intérieure de l'équation des ondes. Private communication, 1998.

[12] K. B. Stolarsky. Algebraic numbers and diophantine approximations. Marcel Dekker, Basel, 1974.

[13] M. Tucsnak. Regularity and exact controllability for a beam with piezoelectric actuator. SIAM J. Control and Optimization, 34:922-930, 1996. 